\title{
The Rotation of Red Giants and Horizontal Branch Stars
}

\author{
J. R. De Medeiros \\ Department of Physics, Federal University of Rio Grande do Norte \\ Campus Universitario 59072-970, Natal, R.N., Brazil
}

\begin{abstract}
.
Rotation is one of the most important observable in stellar astrophysics driving strongly the evolution of stars. This review presents the main observational results on the rotation of Red Giant and Horizontal-Branch stars carried out along the past 50 years. The review brings, in particular, the most recent results based on very accurate measurements of rotational velocity obtained mostly along the last decade.
\end{abstract}

\section{Introduction}

The study of the rotation of evolved stars has as its main challenging goal to understand the stellar angular momentum history once stars evolve through the H-R diagram. Besides, rotation can provide important constraints for models of angular momentum evolution during stellar life and valuable informations on the connection between surface rotation and atmospheric phenomena as well as on tidal interaction, stellar coalescence and engulfing of brown dwarfs and planets. These points indicate, in a broad sense, that stellar rotation is a fundamental problem. Of course, only surface rotational velocity can directly be determined from the observations but there are strong evidences that these rotational velocities are governed largely by the efficiency of angular momentum transport inside the stars.

In spite the fact that stellar rotation has been studied for several centuries, its development is rather slow, in particular for evolved stars. Most of our knowledge on the behavior of the rotation of evolved stars, prior 1980, depends upon the pioneering works by O.Struve and his colleagues and by S. Huang. Early studies include the classic paper by Struve (1930), Herbig and Spalding (1955) and Kraft (1967). These works have just shown how slowly rotating the evolved stars are (Herbig and Spalding 1955; Slettebak 1970). In fact, the pioneering works could only predict upper limits for the rotation rates of evolved stars. Let us recall that until the advent of solid-state detectors, about a quarter of century ago, most of the rotational velocities were measured from blue-wavelength spectrograms by the observation of relatively strong lines or by visual comparison presenting, consequently, poor to modest precision. Kraft $(1965,1970)$ was able to push the limits down to $6 \mathrm{~km} \mathrm{~s}^{-1}$ for the four Hyades giants. In the early 1980's the history on the precision of rotation measurements turned once again when CORAVEL (Baranne et al. 1979; Benz and Mayor 1981) and Furrier analysis (Gray and Endal 1982) showed $v \sin i$ values for giant stars better than $2.0 \mathrm{~km} \mathrm{~s}^{-1}$. Following such developments, we now find in the literature 
rotational velocity measurements for large and homogeneous sample of evolved stars of different luminosity classes from an effort carried out mostly by David Gray at London Ontario University, Frank Fekel at Tennessee State University, Ruth Peterson at University of California at Santa Cruz and, in particular, by Michel Mayor and co-workers at Geneva Observatory.

Evolutionary models taking into account rotation have shown that substantial differences exist between them and their nonrotating counterparts (Endal and Sofia 1979; Gray and Endal 1982; Pinsonneault et al. 1989; Maeder and Meynet 2000). In rotating models there are several additional problems that must be solved in order to explain the stellar evolution. In particular, what is not clear is whether angular momentum is conserved as stars evolve through the Hertzsprung-gap and during the subsequent phases of the evolution, or whether a magnetic braking is needed. Of course, one might also inquire about the nature of rotational discontinuities observed for different luminosity classes. Which behavior rotation should present along the color-magnitude diagram? On the other hand, one might ask how does rotation change in binary stars once stars leave the main sequence and become a giant. Does it follow the same path of single stars, or does it present a different behavior? Another important question concerns the origin of the moderate to moderately high rotation rates observed for giant stars evolving along the horizontal-branch.

Reviews concerning the observational behavior of rotation of evolved stars are rare. Among the last reviews one can underline that by Kraft (1970) and that by Gray (1991), the latter dedicated in particular to subgiants and giants of luminosity class III. In the last IAU event concerning stellar rotation, namely the IAU Colloquium 4, held at the Ohio State University, Columbus, Ohio, 8-11 September 1969, only one work, the review by Arne Slettebak, discussed on the rotation of evolved stars, focusing particularly at the A to F spectral region. Here, I shall focus on the behavior of rotation of red giant stars with masses ranging typically from 1 to 12 solar masses, based on rotational velocity measurements with precisions better than $2.0 \mathrm{~km} \mathrm{~s}^{-1}$, and on the behavior of rotation of stars evolving along the horizontal-branch.

\section{The Rotation of Red Giants in the HR Diagram}

The distribution of rotation of evolved stars in the $v \sin i$ versus $(B-V)$ plan shows a very clear behavior: a discontinuity in $v \sin i$ near $(B-V) \sim 0.55$ (corresponding to $\mathrm{F} 8 \mathrm{IV}$ ) and $(B-V) \sim 0.70$ (corresponding to G0III) for subgiant and giant stars respectively (Gray and Nagar 1985; Gray 1989; De Medeiros and Mayor 1990), and a discontinuity near $(B-V) \sim 0.65$ for bright giants and $\mathrm{Ib}$ supergiants (De Medeiros and Mayor 1990). Stars blueward of the these discontinuities present a wide range of rotational velocity values, from a few $\mathrm{km} \mathrm{s}^{-1}$ to about one hundred of times the solar rotation rate, whereas stars redward of the discontinuities are essentially slow rotators with rotation decreasing smoothly from early-G to late-K types (De Medeiros et al. 1996).

In spite the very clear definition of the discontinuities in the $v \sin i$ versus $(B-V)$ plan, things become more complex when analyzed in the color-magnitude diagram. Figure 1 presents the behavior of the rotation of red giant stars along the H-R diagram, from an analysis by De Medeiros and Mayor (2003). In this 
diagram, evolutionary tracks are from Schaller et al. (1992), whereas luminosities have been derived from Hipparcos parallaxes. The presence of a rotational boundary in the H-R diagram seems clear in this figure. Nevertheless, the location of such rotational boundary depends strongly on stellar mass and, consequently, on luminosity. Let us summarize the main features emerging from this figure:

- There is no discontinuity in rotation for stars with masses lower than about $1.25 \mathrm{M}_{\odot}$. These stars, from the turnoff to the red giant branch (RGB), are essentially slow rotators. Stars with $1.0 \mathrm{M}_{\odot}$, in particular, show typically a solar rotation. Following the approach by Melo et al. (2001) for the angular momentum evolution of $1.2 \mathrm{M}_{\odot}$ stars in M67, these stars probably obey different angular momentum evolution laws on the main sequence and along the RGB: on the main sequence some extra braking is required in addition to angular momentum conservation, whereas along the RGB the angular momentum evolution is controlled by the $I \Omega=$ constant law.

- Stars in the turnoff with masses between 1.25 and $2.0 \mathrm{M}_{\odot}$, typically stars blueward of the rotational boundary, present a wide range of $v \sin i$ values, from a few $\mathrm{km} \mathrm{s}^{-1}$ to about a hundred of times the solar rotation rate, whereas stars with masses between 2.0 and $2.5 \mathrm{M}_{\odot}$ are mostly fast rotators. For stars in the mass range 1.25 to $2.0 \mathrm{M}_{\odot}$ one observes a discontinuity in rotational velocity almost at the same effective temperature region. For stars with mass larger than about $2.0 \mathrm{M}_{\odot}$ the rotational boundary shows a clear tendency to be redward than that for less massive stars.

Now let us move on to the possible root-cause for the behavior of rotation of red giant stars. The main question here is: could the observed behavior in rotation, from the turnoff to the RGB ascent, simply reflect the rapid increase in moment of inertia as the stars evolve along the H-R diagram? Is there a magnetic braking involved? First, calculations by Rutten and Pylyser (1988) and De Medeiros (1990) have shown that for stars with masses larger than $2.0 \mathrm{M}_{\odot}$ the timescale of magnetic braking is higher than the evolutionary timescale into the late-F and early-G spectral regions. Second, models by Endal and Sofia (1979), taking into consideration rigid body rotation, can explain at least the observed mean surface rotational velocity of red giants with masses larger than about $3.0 \mathrm{M}_{\odot}$ at the K spectral region. Third, Gray and Endal (1982) have shown that their model with an uniform specific angular momentum in convective regions can explain the observed surface rotational velocity of the Hyades red giant. The mean rotational velocity of the four Hyades giants coincides with the mean rotational velocity of field giants at the same spectral types or color index (De Medeiros et al. 1996) and mass interval (Pasquini et al. 2000). In addition, the rotational velocities predicted from models by Pinsonneault et al.(1989) are consistent with the observed rotation rate of evolved low mass stars. In short, we cannot escape the conclusion that no loss of angular momentum due to magnetic braking is required to explain the observed mean rotational velocity of red giants with masses larger than about $2.0 \mathrm{M}_{\odot}$. For giant stars with masses shorter than $1.5 \mathrm{M}_{\odot}$ it seems that an additional decline in rotation exists beyond what is expected by the increasing of the moment of inertia. For these stars the timescale of magnetic braking is shorter than the evolutionary timescale into 


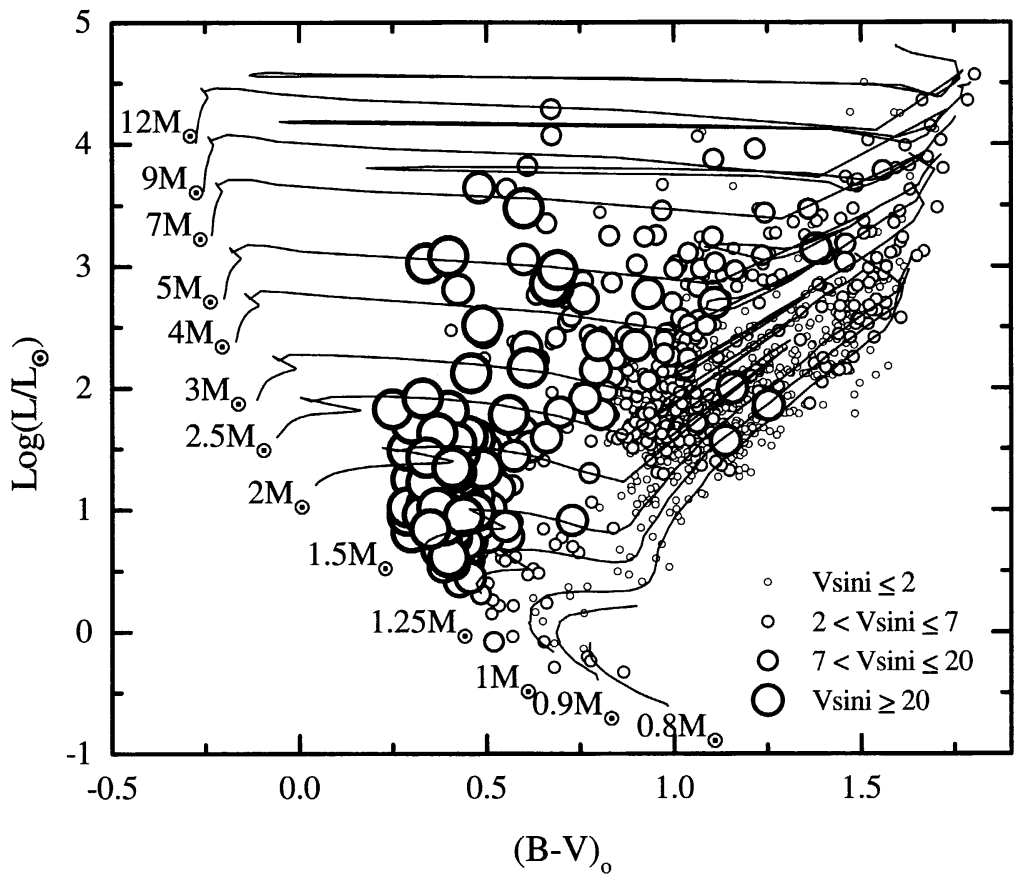

Figure 1. The behavior of rotational velocity for giant stars at the HR diagram (De Medeiros and Mayor 2003), from a sample of about 2000 stars from the turnoff to the red giant branch region.

the late-F and early-G spectral regions (Rutten and Pylyser 1988; De Medeiros 1990).

\section{The Rotational Discontinuity at G5III: An Artifact}

In a series of works, Gray $(1981,1982,1983)$ has claimed a discontinuity in rotation of giant stars near the spectral type G5III, where at least a fivefold drop should occurs, with rotational velocity decreasing from about $25 \mathrm{~km} \mathrm{~s}^{-1}$ to about $5 \mathrm{~km} \mathrm{~s}^{-1}$ (see also Smith 1983). This sudden decline in rotation at G5III is rather surprising. In fact, it is very hard to understand such a rapid braking into the Hertzsprung-gap because evolutionary calculations show that the first crossing evolution of giant stars through this region is very rapid (e.g.: Schaller et al. 1992). 
Before going on let us clear up this confusing point. De Medeiros (1990) and De Medeiros and Mayor (1999) have carried out a robust test to check the origin of the proposed discontinuity at G5III by observing a few G5III stars used by Gray in his analysis. In fact, in the first instance, Gray has combined his $v \sin i$ measurements, obtained with a precision of about $1.0 \mathrm{~km} \mathrm{~s}^{-1}$ with those measured by Alschuler (1975) for which the uncertainty is of about $20.0 \mathrm{~km} \mathrm{~s}^{-1}$. Table 1 compares CORAVEL $v$ sin $i$ measurements from De Medeiros and Mayor (1999) with those values from Alschuler (1975) for the G5III used by Gray for the definition of the discontinuity at G5III. It is clear from this table that, at least for these stars, the $v \sin i$ measurements from Alschuler are unreliable. Consequently, in the present case, one cannot combine high resolution $v \sin i$ with those from Alschuler(1975) for any scientific purpose. If one removes the Alschuler's G5III stars used by Gray, there will be only one G5III star with a significant rotation rate in the region of the claimed discontinuity at G5III. This star is HD 153751 a spectroscopic binary with orbital period of 39.8 days, zero eccentricity and a $v \sin i$ of $24.0 \mathrm{~km} \mathrm{~s}^{-1}$. Such a high rotation is very probably due to synchronization effects. In addition, Gray in his early works did not observe stars blueward of G5III, which have introduced a strong bias in the behavior of rotation as a function of colors. In short, the discontinuity at G5III was just the result of a bad combination of data made by Gray, which, associated with a selection effect, led him erroneously to define such a discontinuity.

Table 1. Comparison between CORAVEL and Alschuler (1975) $v \sin i$ measurements in $\mathrm{km} \mathrm{s}^{-1}$ for giant stars from De Medeiros and Mayor (1999)

\begin{tabular}{cccc}
\hline HD & ST & $\begin{array}{c}v \sin i \\
(\text { COR })\end{array}$ & $\begin{array}{c}v \sin i \\
\text { (Alschuler) }\end{array}$ \\
\hline 5137 & G5III & 2.0 & 22 \\
97561 & G5III & 3.8 & 21 \\
127742 & G5III & 5.9 & 26 \\
210264 & G5III & 5.0 & 27 \\
\hline
\end{tabular}

\section{The Rotation of Binary Systems with Red Giant Components}

Tidal theory (e.g.: Zahn 1977) predicts that, in late-type binary systems, viscous dissipation of time-dependent tidal effects should produce synchronization between rotation and stellar orbital motion, as well as circularization of the orbit of the system. A solid way to test such effects consists in the determination of precise rotation rates for a large sample of late-type binary systems with giant components presenting a wide variety of values of orbital parameters. To establish the behavior of rotation as a function of effective temperature, spectral type and $(B-V)$ color index and the link between rotation and tidal effects in binary systems with red giant components, De Medeiros et al. (2002) have analyzed the rotational velocity for a large sample of 134 such binary systems, along the spectral region from middle $\mathrm{F}$ to middle $\mathrm{K}$. As shown in Fig. 2, the distribution of rotational velocity for binary systems with red giant components seems to 
follow the same behavior of their single counterparts, with a drop around G0III. Blueward of this spectral type there is a trend for a large spread in the values of rotational velocity, from a few kilometers per second to at least $40 \mathrm{~km} \mathrm{~s}^{-1}$. Redward of G0III there are a considerable number of binary systems with moderate to moderately high rotation, all these systems presenting orbital period shorter than about 250 days and circular or nearly circular orbits. This seems to reflect, clearly, the effects of synchronization between rotation and orbital motions predicted by tidal theory. Except these synchronized systems, the large majority of binary systems with a red giant component of spectral type later than G0III are composed of slow rotators. Whereas the work by De Medeiros and co-workers is devoted to stars of population I, more recently Carney et al. (2003) have found indications that high rotation in metal-poor binary systems is very probably induced by tidal locking between rotation and orbital motions.
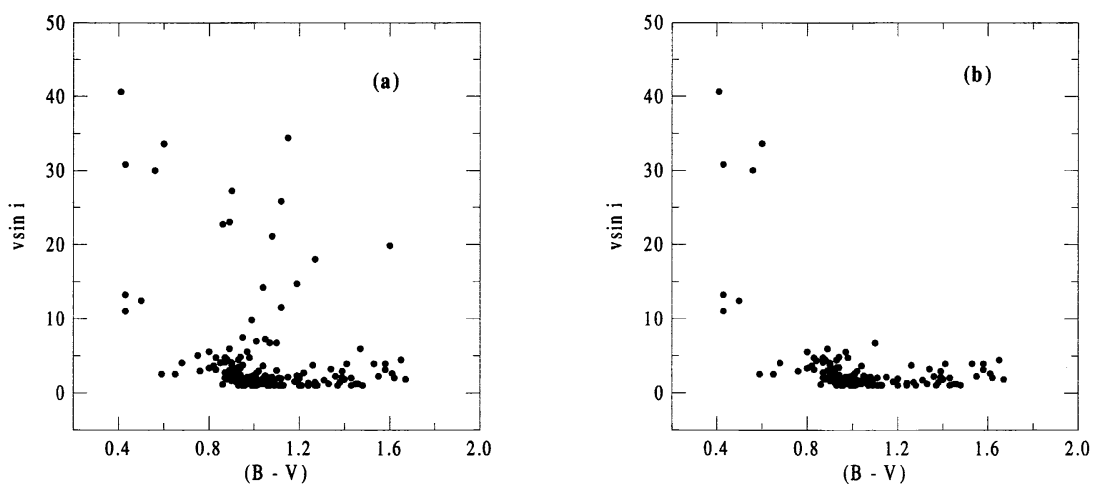

Figure 2. Distribution of the rotational velocity $v \sin i$ for binary systems with red giant components (De Medeiros et al. 2002). (a) For all the binary systems. (b) For systems with G- or K-type component but with orbital period longer than 250 days and all the systems with F-component.

\section{The Rapidly Rotating Red Giant}

While the vast majority of single red giant are slowly rotating stars, a growing list of such stars violates this rule presenting moderate to fast rotation (Fekel et al. 1986; Balona 1987; Fekel and Balachandran 1993; Fekel 1997; De Medeiros and Mayor 1999). The evolutionary status of these stars can be considered essentially unknown in spite a number of claims by different authors. The first claim was that they were binaries before the components coalesced, following the evolutionary status proposed for the FK Comae stars, which may be descendants of W UMa binaries whose components have merged (Bopp and Stencel 1981; Bianchi et al. 1985). Simon and Drake (1989) have suggested that the rapid rotation of these stars may be the result of a sudden dredge-up of angular momentum from the interior. Other interesting mechanisms that might be responsible for the enhanced rotation of these stars are: the absorption of orbiting planets and the accretion of brown dwarfs by the giant stars (e.g. Siess and Livio 1999). More recently, Carney et al. (2003) have re-explored the latter scenario 
and have shown that the capture of a planetary-mass companion as a red giant expands in radius could explain the high rotational velocity of metal-poor red giant stars.

\section{The Rotation of Horizontal-Branch Stars}

Since the pioneering works by Peterson (1983, 1985a, 1985b), rotational velocity of horizontal-branch stars with temperatures between 7000 and $28000 \mathrm{~K}$ have been measured in the field and in globular clusters (Peterson et al. 1995; Cohen and McCarthy 1997; Behr et al. 2000). More recentely Recio-Blanco et al. (2002, 2003) have presented rotational velocity for 61 stars in the extended blue horizontal-branches of four Galactic globular clusters. Behr et al. (2000) suggested the existence of a discontinuity in the distribution of rotational velocity versus effective temperature at $T_{\text {eff }} \sim 11000 \mathrm{~K}$. The data of Recio-Blanco et al. (2002, 2003) also reveal clearly a similar discontinuity. In short, stars with $T_{\text {eff }}$ between 11000 and 8000 show a range of rotation rates between a few kilometers per second and $40 \mathrm{~km} \mathrm{~s}^{-1}$, whereas stars hotter than about $11000 \mathrm{~K}$ present $v \sin i$ lower than about $15 \mathrm{~km} \mathrm{~s}^{-1}$. Rotational velocities from Kinman et al. (2000) and Da Silva (2003) for field horizontal-branch stars show the same behavior observed for globular cluster stars.

Figure 3 presents the distribution of $v \sin i$ versus effective temperature based on a combination of data of Peterson et al. (1995), Behr et al. (2000), Recio-Blanco et al. (2003) for horizontal-branch stars of globular clusters, and from Kinman et al. (2000) and Da Silva (2003) for field horizontal-branch stars. The wide range of $v \sin i$ for stars located between 8000 and $11000 \mathrm{~K}$, with a clear discontinuity for stars of globular clusters near $11000 K$ is quite impressive. The usual explanation for the increase in rotational velocity between the main sequence and the horizontal-branch is that the cores of the main sequence stars retain a substantial amount of their primordial angular momentum and are rotating rapidly. The horizontal-branch region would be populated by stars which have undergone extreme mass loss at the RGB and thus that high angular momentum should become evident at the surface and appear as higher $v \sin i$. New models by Sills and Pinsonneault (2000) suggest that the observed range in rotational velocity on the horizontal-branch is caused by internal angular momentum redistribution, which occurs on a timescale comparable to the evolution of the stars on the horizontal-branch. Nevertheless this model does not predict the discontinuity in rotation at $T_{\text {eff }} \sim 11000 \mathrm{~K}$.

More recently, Carney at al. (2003) and Da Silva (2003) have found signs of significant rotation in metal-poor single red giant and single red horizontalbranch field stars, with values of $v \sin i$ exceeding $12 \mathrm{~km} \mathrm{~s}^{-1}$. As discussed by Carney and co-workers, the absorption of a planet as the red giant expands in radius could explain the high rotational velocity observed for these stars.

\section{Summary}

In the thirty-four years since the last IAU meeting dedicated to stellar rotation, an important number of conclusive studies (fortunately!) enlarged the frontiers of our understanding on the fascinating world of this fundamental parameter. 


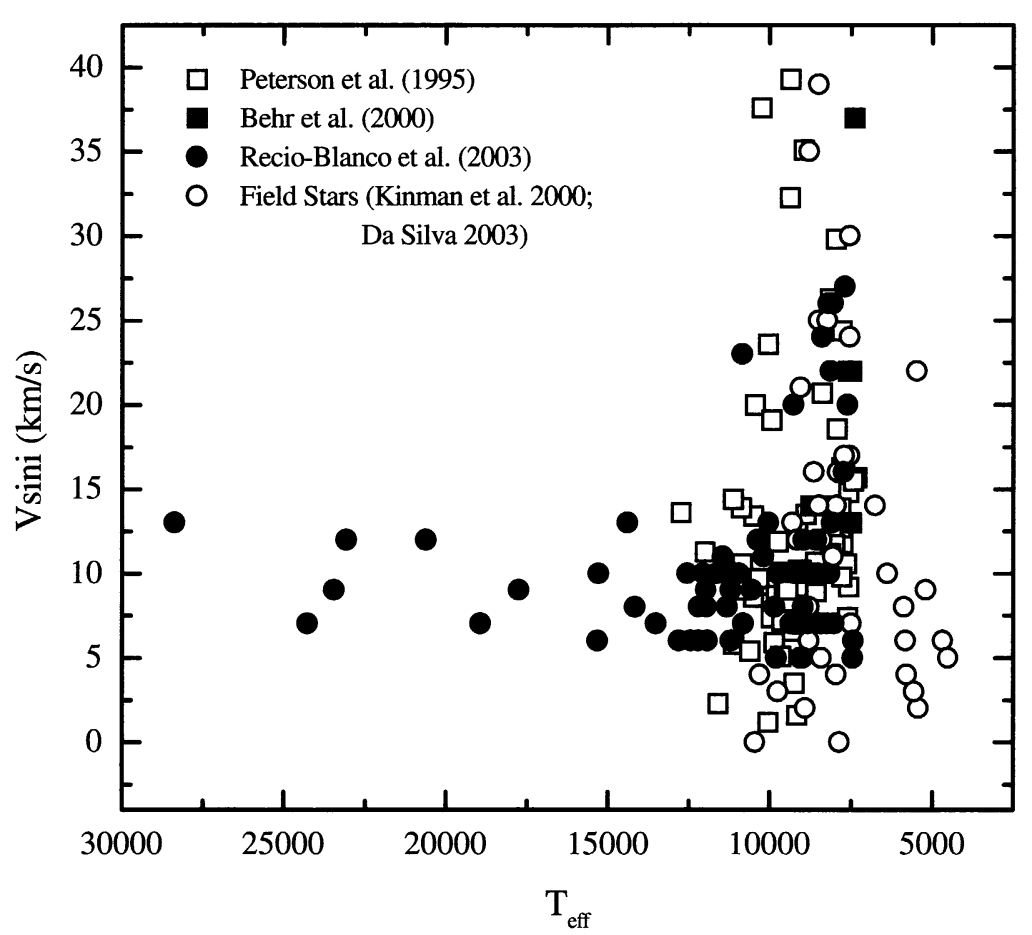

Figure 3. The distribution of rotational velocity for giant stars at the horizontal-branch, as a function of the effective temperature.

But, an important number of unresolved issues (unfortunately!), associated with an increasing number of new open questions (fortunately!), revolve around the nature and evolution of stellar rotation.

The observational pattern of rotation for single and binary red giant stars is now well established, with a clear rotational boundary along the HR diagram for single stars with masses larger than about $1.25 \mathrm{M}_{\odot}$, its location depending clearly on stellar mass. For stars with masses larger than about $2.0 \mathrm{M}_{\odot}$ there is no convincing evidence for magnetic braking. Stars with masses around $1.5 \mathrm{M}_{\odot}$ present some evidence for loss of angular momentum due to magnetic braking but the extent of this mechanism is not yet clear. Stars with masses lower than $1.25 \mathrm{M}_{\odot}$, from the turnoff to the RGB, are essentially slow rotators. These stars probably obey different angular momentum evolution histories, with some extra braking on the main sequence and an evolution along the RGB controlled the $I \Omega=$ constant law.

Regarding binary systems, there is now compelling evidences for synchronization between rotation and orbital motions in solar and metal-poor red giant 
stars. Binary systems with orbital period shorter than about 250 days are mostly in synchronization, presenting enhanced rotation in relation to their counterparts with orbital period larger than 250 days.

Among the most puzzling open questions are those concerning the rootcause of the enhanced rotation in a few single red giant stars, as well as in stars evolving along the horizontal-branch. The nature of the discontinuity in rotation of horizontal-branch stars at $T_{\text {eff }} \sim 11000 \mathrm{~K}$ is another puzzle that should lead to new observational and theoretical efforts.

The past century have produced a great deal of informations on the surface rotation of stars. Now, it seems to be time to disentangle the details on the behavior of rotation in the different regions of the HR diagram. It is also time to move toward the interior of stars looking for differential rotation which may represents the main key for our complete understanding of the evolutionary history of angular momentum in stars. The door is open for this latter relevant topic with the pioneering discovery of solar-like oscillations in the G7 giant star $\xi$ Hya by S. Frandsen and colleagues (2002)!

I dedicate this work to my kids Leonardo and Edoardo who make my happiness go spinning along and to Professor Michel Mayor who pointed me to the beauty of the Stellar Rotation. Thanks Michel! It is my pleasure to thanks all my friends at Geneva Observatory who made my many years lived in Geneva pleasant and productive. It is also my pleasure to thanks Andre Maeder and Philippe Eenens for organizing such a stimulating meeting. I am grateful to $C N P q$ and CAPES brazilian councils for continued financial support and to Bruno Canto Martins for his support in the preparation of this paper.

\section{References}

Alschuler, W. R. 1975, ApJ 182, 809

Balona, L. A. 1987, SAAO Circ., 11, 1

Baranne, A. Mayor, M. and Poncet J. L. 1979, Vistas Astron., 23, 279

Behr, B. B., Djorgovski, S. G., Cohen, J. G., McCarthy, J. K., Cote, P., Piotto, G. and Zoccali, M. 2000, ApJ 528, 849

Benz, W. and Mayor, M. 1981, A\&A 93, 235

Bianchi, L., Grewing, M. and Kappelmann, N. 1985, A\&A 149, 41

Bopp, B. W. and Stencel, R. E. 1981, ApJ 247, L131

Carney, B. W., Latham, D., Stefanik, R. P., Laird, J. B. and Morse, J. A. 2003, AJ 125, 293

Cohen, J. G. and McCarthy, J. K. 1997, AJ 113, 1353

Da Silva, J. R. P. 2003, Ph.D. Thesis, Federal University of Rio Grande do Norte

De Medeiros, J. R. 1990, PhD Thesis, Geneva Observatory

De Medeiros, J. R. and Mayor, M. 1990, in Cool Stars, Stellar Systems and the Sun, Ed. G. Wallerstein (ASP, San Francisco), p. 404

De Medeiros, J. R., Da Rocha, C.and Mayor, M. 1996, A\&A 314, 499

De Medeiros, J. R. and Mayor, M. 1999, A\&AS 139, 433

De Medeiros, J. R. and Mayor, M. 2003, in preparation

De Medeiros, J. R., Da Silva, J. R. P. and Maia, M. R. G. 2002, ApJ 578, 943

Endal, A. and Sofia, S. 1979, ApJ 232, 531 
Fekel, F. C., Moffet, T. M. and Henry, G. W. 1986, ApJS 60, 551

Fekel, F. C. and Balachandran, S. 1993, ApJ 403, 708

Fekel, F. C. 1997, PASP, 109, 514

Frandsen, S., Carrier, F., Aerts, C., et al. 2002, A\&A L5

Gray, D. 1981, ApJ 251, 155

Gray, D. 1982, ApJ 262, 682

Gray, D. 1983, PASP, 95, 181

Gray, D., and Nagar, P. 1985, ApJ 298, 756

Gray., D. 1989, ApJ 347, 1021

Gray, D. 1991, in Angular MomentumEvolution of Young Stars, Ed. S. Catalano and

J. R. Stauffer (Kluwer Academic Publishers), P. 183

Gray, D. and Endal, A. S. 1982, ApJ 254, 162

Herbig, G. H. and Spalding J. F. 1954, ApJ 121, 118

Kinman, T., Castelli, F., Cacciari, C., Bragaglia, A., Harmer, D. and Valdes, F. 2000, A\&A 364, 102

Kraft, R. 1965, ApJ 142, 681

Kraft, R. 1967, ApJ 150, 551

Kraft, R. 1970, in Spectroscopic Astrophysics, ed G. H. Herbig (Berkeley and Los Angeles: University of California Press), P. 385

Maeder, A. and Meynet, G. 2000, Annu. Rev. A\&A 38, 143

Melo, C. H. F., Pasquini, L. and De Medeiros, J. R. 2001, A\&A 375, 851

Pasquini, L., De Medeiros, J. R. and Girardi, L. 2000, A\&A 361, 1011

Peterson, R. C. 1983, ApJ 275, 737

Peterson, R. C. 1985a, ApJ 289, 320

Peterson, R. C. 1985b, ApJ 294, L35

Peterson, R. C., Tarbell, T. D. and Carney, B. W. 1983, ApJ 265, 972

Peterson, R. C., Rood, R. T. and Crocker, D. A. 1995, ApJ 453, 214

Pinsonneault, M. H., Kawaler, S. D., Sofia, S. and Endal, A. 1989, ApJ 232, 531

Sills, A. and Pinsonneault, M. H. 2000, ApJ 540, 489

Recio-Blanco, A., Piotto, G., Aparicio, A. and Renzini, A. 2002, ApJ 572, L71

Recio-Blanco, A., Piotto, G., Aparicio, A. and Renzini, A. 2003, Private communication

Rutten, R. G. M. and Pylyser, E. 1988, A\&A 191, 227

Schaller, G., Schaerer, D., Meynet, G. and Maeder, A. 1992, A\&AS 96, 269

Siess, L. and Livio, M. 1999, MNRAS 304, 925

Simon, T. and Drake, S. A. 1989, ApJ 346, 303

Slettebak, A. 1970, in Stellar Rotation, IAU Colloq. 4, Ed. A. Slettebak(Gordon and Breach Science Publishers), p.3

Smith, R. C. 1983, Nature 303, 130

Struve, O. 1930, ApJ 72, 1

Zahn, J.-P. 1977, A\&A 57, 383 